\title{
Intelligent Wheelchairs and Assistant Robots
}

\author{
Josep Amat \\ Institut de Robòtica industrial (IRI)- CSIC/UPC \\ Barcelona (SPAIN) \\ Amat@esaii.upc.es
}

\begin{abstract}
This work presents an overview over the main technological aids oriented to the rehabilitation of the physically disabled so that they can get some independence. These aids range from wheelchairs up to the assistant robots developed in the last years
\end{abstract}

\section{Introduction.}

Technological developments in the last years have not only allowed an increase of the automation level in industry, they have also enabled the manufacture of many consumer goods. Such goods considered by society as basic elements to improve the quality of life, are equipments such as automobiles, audio and video devices, electric appliances or even personal computers.

Many of these equipments, direct or adequately modified can also constitute valuable aiding elements to people with physical disabilities produced by diseases, accidents or also to persons with physical limitations due to old age.

The current availability of equipments that constitute today valuable aids for disabled people, come from an increasing number of specialized companies that respond to the needs of a demand more and more important. On the other side, these companies have available an increasing range of components and devices originally addressed to industrial automation, but that, conveniently adapted, also allow the automation of lighting systems, ventilation, and access to or manipulation of the most common home elements. These elements start to be used by persons that suffer from important deficiencies in their motor capabilities [1].

The companies specialized in providing technological equipment to physically disabled persons to increase their independence, offer everyday new technological resources, ranging from new materials to robotics. These companies also rely on the support of many research centers, making possible with this cooperation to significantly increase the market supply of this kind of aids and to appreciably reduce their cost. Thus, more potential users can reach these products, fig. 1 .

\section{Technological aids for mobility}

One of the most usual disabilities of old people is their lack of mobility. This disability make them unable not only to move outside, in public thoroughfare, but even to carry out the necessary short runs at home.

The use of wheelchairs, that started to be developed at the beginning of this century, has enabled to provide a very efficient solution to the need of mobility, 
without any other external aid, provided the impaired person can use his or her upper limbs.

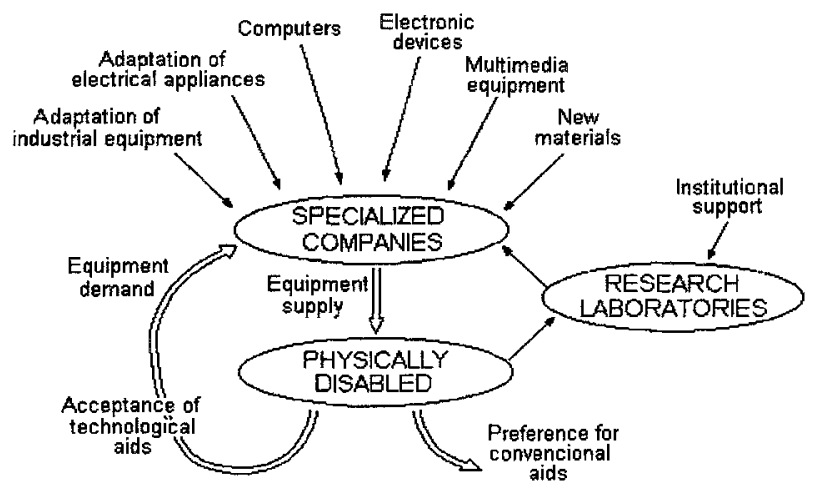

Fig. I Impact of technological advances in the supply of aids for disabled.

The use of wheelchairs has expanded enormously in the second half of this century, but only recently, in the last years, they have started to be motorized. Consequently, they also can be used by people with motor impairment in their upper limbs, severely enough to impede their manual propulsion. It is specially the development of technologies tied to automation and even those developed for mobile robots, what have allowed to build wheelchairs endowed with a control unit to facilitate its driving. With these more advanced wheelchairs, even people with very low remaining motion in their hands can drive them without any external aid.

Nowadays, motorized wheelchairs can be driven by means of intelligent controllers. These are microcomputer based controllers enabling the chair to carry out straight line trajectories independently of terrain irregularities, to change direction incrementally or to rotate in place, fig.2. Each user needs his own adapted interfaces, according to his deficiency, to control the wheelchair [2]. For instance, these interfaces could be adapted to detect the head movements when the user can not use his hands.

With the aim to control the trajectories with the support of a microcomputer, these wheelchairs are endowed with angular encoders in their steering wheels. The encoders supply the data required to measure runs of the order of millimeters, and consequently it is possible to calculate, at every moment, the wheelchair trajectory and adjust it to the set points given by the user. This procedure allows to detect the wheelchair deviations produced by the terrain unevenness or its irregularities, and to compensate them. In the same way, it allows to memorize trajectories and to perform automatically their inverse runs. This facility allows to go out from constrained spaces, avoiding the great amount of maneuvers that would be necessary driving the chair manually. This is the case, for instance, of leaving a narrow bathroom or an elevator.

These intelligent wheelchairs are still little accepted by users, but not due to their cost, since the additional cost of electronics with respect to the cost of the motorized wheelchair is not very relevant. Acceptability is limited by a technological factor, that still predispose negatively most of the potential users. 
Such intelligent controllers can also benefit from other advances reached in the field of autonomous navigation or mobile robots [3]. For instance, endowing the wheelchair with absolute positioning systems, GPS type, or with obstacle detection systems. Such systems enable to have available motorized wheelchairs with aids for navigation in urban environments, even for the severely disabled.

The efforts carried out with the aim to endow wheelchairs with the possibility to go up and down stairs has achieved, technically speaking, positive results, fig. 3. But, their high cost and their bulkiness, together with the availability of other existing aids to go up vertical unevenness, has had as a consequence that these kind of wheelchairs are scarcely used.
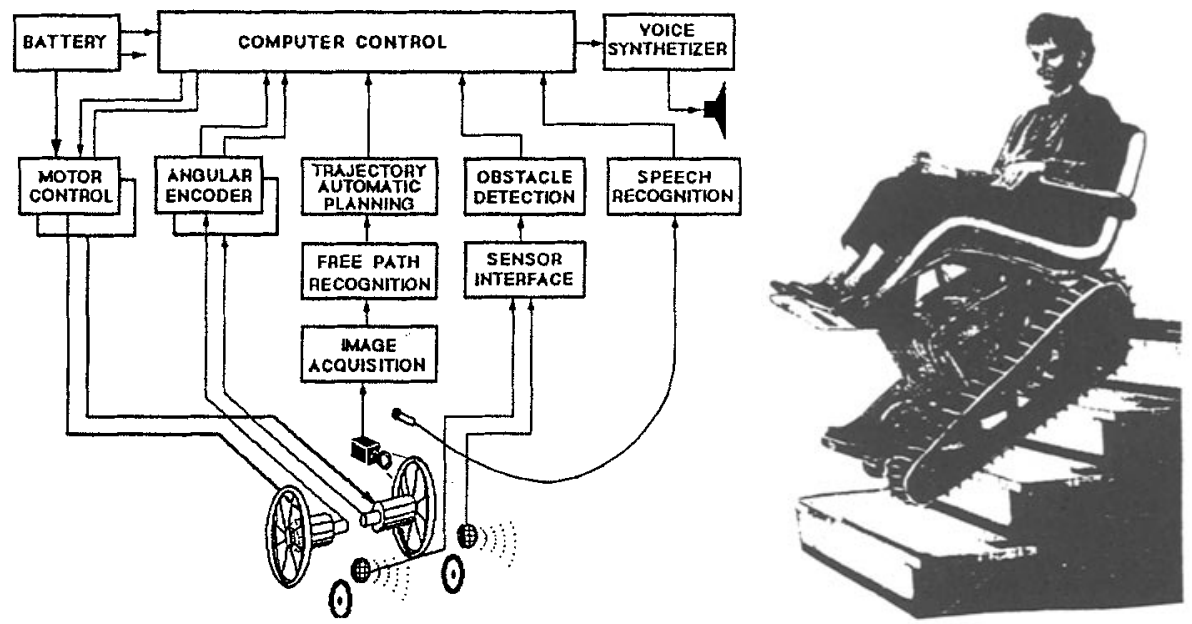

Fig. 2 Control structure of an intelligent wheelchair

Fig. 3 Wheelchair with caterpillar to up and down stairs

On the other hand, some efforts have been done in the design of new chairs and the study of the materials to use, to build wheelchairs adequate to practice some kind of sports or physical activities. Nowadays, with very light wheelchairs having an ergonomic design, it is possible to practice athletics, basketball, cycling or even to fly with delta wings, fig. 4 .

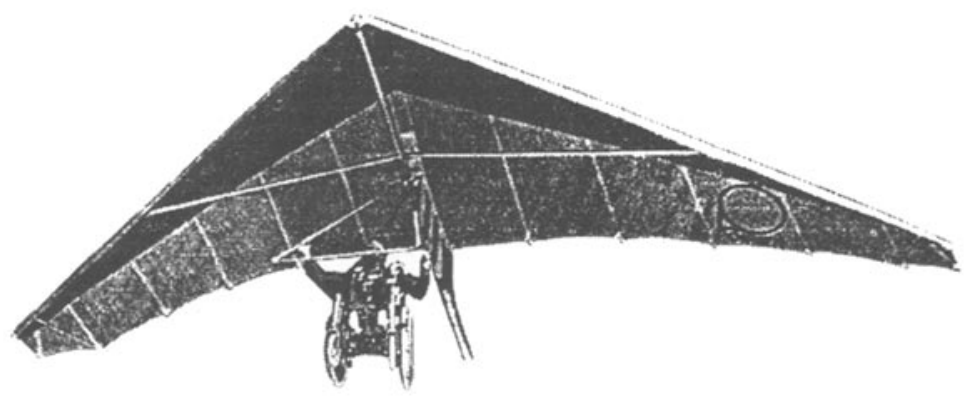

Fig. 4 Possibility for a disabled to practice sports 


\section{Prosthetic elements}

Robotics advances are also applicable to the development of aids oriented to the rehabilitation of people with muscular atrophy or amputees, which, due to this impairment have missed the mobility of one or more of their limbs.

The development of arms and legs, either prosthesis or orthesis, started in the seventies and has already attained remarkable performances. With current technology it is possible to build arms or legs with a physical appearance, totally mimetic to human's. This fact makes them, from this point of view, totally acceptable. A technological difficulty, presently, comes from the power supply requirements, specially for lower limbs which consumption is far higher. On the other side, another difficulty is due to their control, according to the user's will, every moment of his daily life.

When the physical deficiency comes from a recent amputation or even from a muscular atrophy, it can be possible to control the joints of a prosthetic arm using the user own myoelectric signals, those generated by the brain to activate the muscles in healthy people [4]. In this case the myoelectric signals are acquired by means of electrodes, are amplified and afterward processed to identify the kind of movement desired by the user. Fig. 5 .

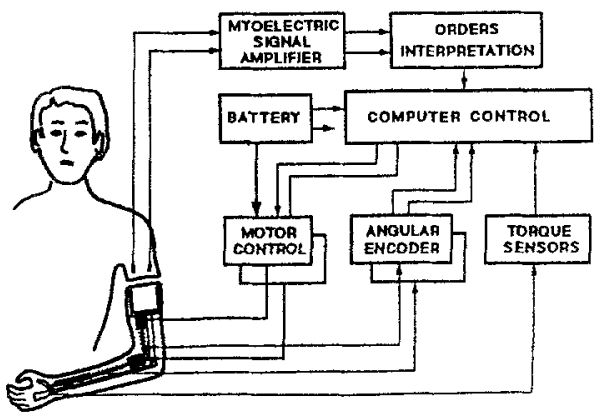

Fig. 5 Schema of the control system of a prosthesis by means of myoelectric signals

Some of the problems not still solved are the capabilities to differentiate signals from noise, and to interpret many orders given by the brain. Currently, only the most significant orders are interpretable, orders such as up and down, approach and retrieve the arm or open and close the hand. Imprecision in the interpretation of these orders forces the user of such devices to initiate a process of learning his new capabilities, which though they are very few with respect to a healthy arm, enable the user to recover an important level of his autonomy.

When the physical impairment affects also the generation of the myoelectric signal, being either due to an injury in the spinal cord or in the brain itself, it is necessary to foresee an adapted interface. This interface activated by the user's remaining movements (head, face or mouth movements) constitutes the means of communication with the computer in order to control the movements of the arm until the desired actuation is attained.

Apart of the situations in which the motorized prosthesis are indispensable, as in the case of amputees of the two arms, their acceptability is still very low, even considering the degree of perfection attained in their aesthetics. The Waseda hand, 
built at Waseda University [5] in 1985 offers an excellent similarity to the human skin, fig. 6, but the servitude that the weight of its power supply represents, and the noise produced by its motors to execute the hand movements are important problems. For these reasons, in many cases, orthopedic passive arms are preferred. The geometry of such arms is configurable by the user, which utilizes this orthopedic arm as a complement to his healthy arm. He learns to modify the position of the artificial arm with almost imperceptible movements of his arm or body in order to reach the best position of the prosthesis for each task to carry out.

The obtention of satisfactory use of robotized arm prosthesis, makes it necessary to still perform research efforts in the design of low size actuators, more efficient and noiseless, as well as in the development of more intelligent control systems.

In this line some other prototypes have been developed. Among them we can mention the "Sams" hand (Southampton Adaptive Manipulation Scheme) developed in 1994. This hand controls independently the movements of the forefinger and the thumb from the other three fingers. This increase of degrees of freedom provides a higher versatility of the hand movements. This greater versatility carries with it at hierarchical control structure in which every joint has available a specific controller that receives also information from the force and sliding sensors available in the hand. These controllers are coordinated by a higher level controller that executes and supervises the actuation programmed by the user, from high level orders that enable to attain a more intelligent control .

Another procedure followed to recover the movement of upper limbs in case of muscular atrophy, in which the motor capability is lessened in such a way that the muscles can not even hold the weight of the own arm, consists in the use of an exoskeleton. In this case, the control of the joints from the endowed force sensors, enable to compensate the weight of the arms and to utilize the user's remaining movements to attain the exoskeleton desired movements.

When the degree of atrophy does not allow to detect the movements the user wishes to perform, it is necessary to use an adequate interface, operating either from orders given through the head movements or orally, using a voice recognition system.
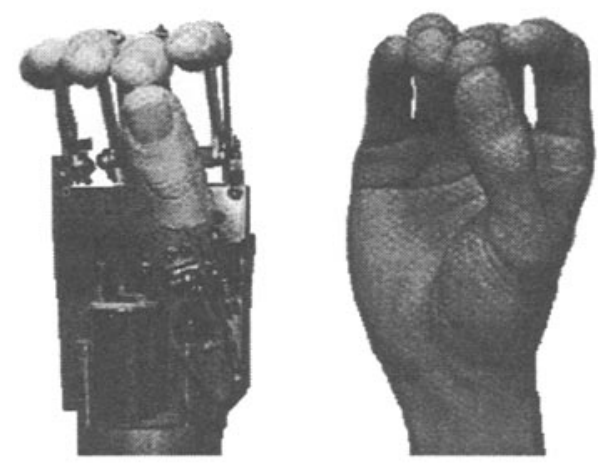

Fig. 6 Prosthetic hand with human appearance built at Waseda University

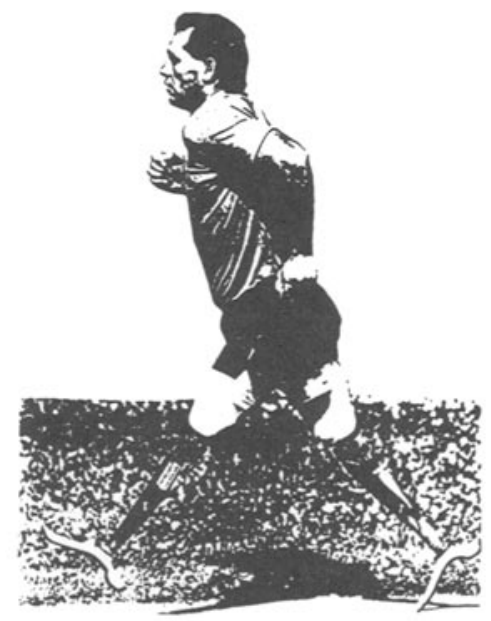

Fig. 7 Mobility attained using orthopedic legs 
The development of lower limb prosthesis presents the additional drawbacks of requiring a power supply of higher capacity, and the fact of making the generated locomotion movements compatible with the body balance. Some prototypes have already been designed, able to execute a sequence of movements with an orthopedic leg, from the movements given by the not injured leg while walking over a flat soil, or even climbing or descending stairs.

In the same way, the coordination of two orthopedic legs has also been attained. The generation of a sequence of movements enabling the user to walk or to run, fig. 7 , has also been proved [6]. Nevertheless, this kind of prosthesis have still more difficulties to reach its acceptability, in front of the solutions based on the use of wheelchairs.

\section{Assistant robots}

The possibility to rely on the use of robots to aid a disabled to get certain independence, even to severely disabled such as tetraplegic, was considered in the eighties. The Veterans Administration Medical Center had available already in 1986 an advanced assistant robotic prototype.

The goal of such robots is to replace the lack of motion capability of an impaired person to be able to approach and to manipulate objects in his environment, without the need to continuously rely on an assistant. And even, to be able to perform autonomously a certain number of daily life activities, such as eating grooming or toileting.

Basically, three different kind of assistant robots can be considered: those mounted over the user own wheelchair; those installed fixed close to the user, and those installed over a mobile base, to be able to move within a limited environment.

\subsection{Assistant robots mounted on the wheelchair}

The advantage of having a robotic arm mounted on the own wheelchair is that the user can move freely and use his auxiliary arm to manipulate objects at any place in his home. But, on the other hand, this option has the drawback of requiring to always transport this device with its significant volume and weight. Fact that sometimes can limit the user's accessibility.

Probably, among all the robots installable on wheelchairs, the one that has attained a certain acceptance is "Manus" developed by TNO in the Netherlands, from 1981 to 1990, in the frame of a European project within the TIDE program [7]. The robot was commercialized in 1991. This robot, fig.8, has seven degrees of freedom, and has been designed so as to attain a great accessible area, being even able to get objects from the floor. Its cylindrical structure with a telescopic base is actuated by means of electrical actuators. The arm can be folded so as to minimize the occupied space when not in use. The control is performed by means of a joystick and with the aid of a simplified functions keyboard its use is much simpler, even performing relatively complex tasks.

Its acceptance and diffusion in Europe, as well as in other countries around the world, has enabled to organize a user's group to facilitate the interchange of experiences among users with different remaining motions. The gained experience allows to solve some of the problems detected and to improve its performances. 


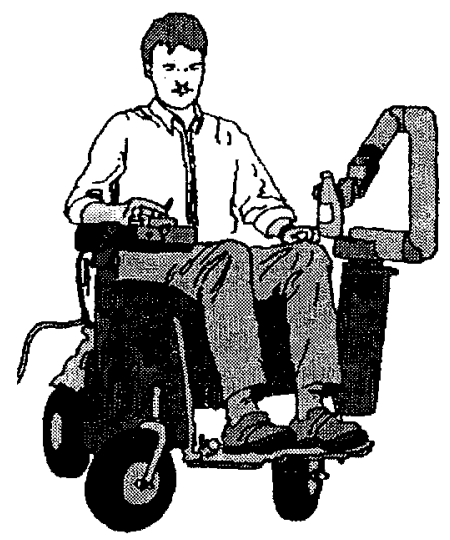

Fig.8 The Manus assistant robot.

Another prototype of robotic arm mounted over a wheelchair is "Inventaid" developed by the Papworth group in 1992. This robot has six degrees of freedom and is moved by means of pneumatic actuators. Its control system is very elemental, having the user to control the movements joint by joint. This structure represents a major simplicity of the control system, but requires a higher user's ability. But, the experience has shown, that with an adequate training, it is possible to perform efficiently very different kind of tasks, having certain complexity. These results have been obtained by users motivated by the utilization of this kind of technological aids.

On the other hand, the use of pneumatic technology limits the force that the arm can perform, and even the robotic arm can manipulate objects up to $4 \mathrm{Kgs}$., it is very adequate and safe to operate very close the user.

Another robot with these characteristics is "Magpie" [8] developed in cooperation by Oxford Orthopedic Engineering and Nulfield Orthopedic Center in 1994. It is a powerless articulated arm, that is, the movement of the arm are obtained from the actuation of some part of the user's body, such as the head or a foot, and the propagation of these actions through simple mechanical transmissions. This mechanical arm, is more limited, but allows to perform tasks such as approach objects, or to feed oneself without external aids.

\subsection{Stand alone robots}

This kind of assistant robots have been conceived to operate very close to the user, but since they are installed on a fix base, independent from the wheelchair, they do not have any weight or consumption constraint. Therefore, these robots can count on any kind of peripheral devices to provide a higher versatility and more intelligence to the robot interface for its control. Thus, the robotic unit can be provided with a vision system designed to guide the robot towards the user, from more precise orders, or a vision system to locate and to recognize the most frequent elements in the environment. It could also be provided with a voice recognition and a voice synthesizing system or the adequate interface to control other elements of its environment [9], fig. 9. 


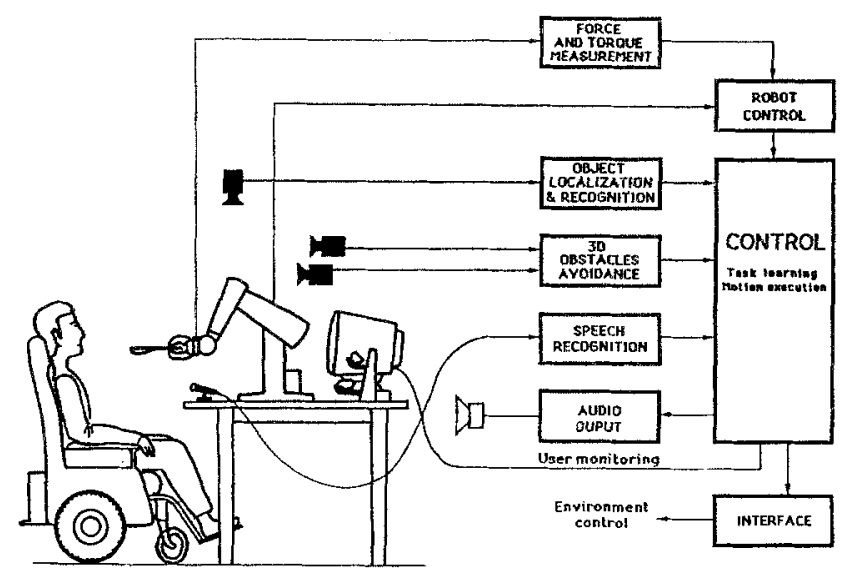

Fig. 9 General structure of an assistant robot

One of these robots is Tou, developed at the Universitat Politècnica de Catalunya from 1989 to 1992 . It is characterized by its soft structure, to guaranty the user safety and at the same time to offer a more friendly presence and touch to the user [10].

Its architecture is constituted by a set of cylindrical shaped foam rubber deformable modules: Each cylinder has two pairs of antagonistic wires, that produce its deformation in two orthogonal directions, actuated by electrical motors. This architecture enables to deform the arm to obtain the adequate curvature for the end effect to reach the point desired by the user, fig. 10.

This robot has available different kind of interfaces, according to the user's needs, to interpret a set of basic orders that are: a voice recognition system, an adapted keyboard or a joystick. A vision system aids the robot guidance towards the objects of the environment for their grasping. Since the robot flexible structure carries with it a high imprecision in its movements, this guiding support facilitates the user's arm guidance.

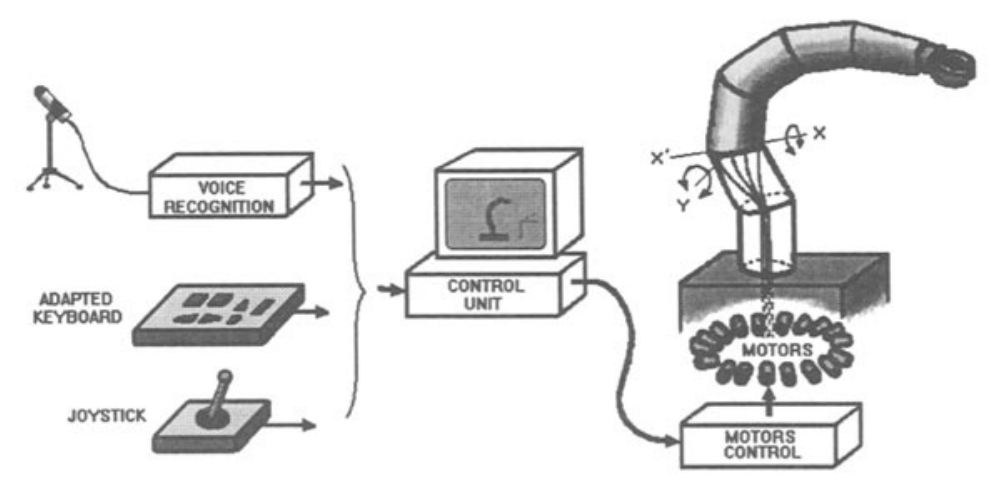

Fig.10 Structure of Tou 
Another kind of stand-alone robots, even it is endowed with wheels to move it more easily when necessary beside the user is "Handy". This assistant arm was developed at the University of Keele [11] in 1993 and has been successively improved. It is mainly oriented to feed the disabled user. Its movements are pre-programmed and the user can fix his or her own rhythm to bring the food from the plate to the mouth, with an interface adapted to his remaining movements.

The assistant robot "Isac" (Intelligent Soft Arm Control) constitutes another aid with these performances. It was developed at the University of Vanderbilt (1991) and manufactured afterwards in Japan. This arm is pneumatically actuated and it is constituted by inflatable elements, equivalent to inflatable muscles, called rubbertuators [12].

This arm is also provided with a vision system that corrects the trajectory towards the different objects on a table, to facilitate user's operation.

\section{3 Assistant robots on a mobile base}

To increase the arm accessibility without requiring a too big structure that predisposes negatively the user to utilize it at home or at work, other projects have been developed providing the robot with mobility within the required work space, using either rails or mobile platforms.

The prototype developed at the Department of Veterans Affairs in Palo Alto, (CA) in 1986 is DEVAR (Desktop Vocational Assistant Robot). It consists of a Puma-260 robot [13] installed on rails, thus reaching a wide working area. Fig. 11.

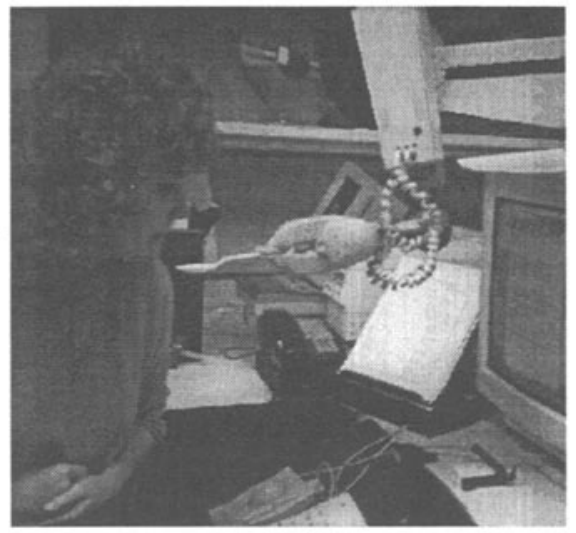

Fig.11 The Devar workstation

This arm is controlled with a joystick and the computer keyboard if the user's remaining movements are enough, or by voice. The robot can either approach food to the user's mouth or to take papers from a file of books from a shelter. It manipulates a CD or can use the microwaves to heat food or to approach some pills.

RAID (Robotic for Assisting the Integration of the Disabled) is the European alternative to DEVAR, developed in the frame of the TIDE program and coordinated by Armstrong Ltd. UK. The main robotic tasks are the manipulation of office objects, documents, books, diskettes located in the supports designed for the workstation. The robot, the system and the wheelchair are controlled by the same interface located in the wheelchair [14]. 
In spite of the successive improvements of the initial prototypes and the attained effectiveness, these two systems are not yet commercial products due to their high cost and the environment complexity.

Another version of assistant robot developed by the Veterans Administration R\&D Center in 1988 is MOVAR (Mobility Vocational Robot) that consisted in installing the same Puma robot on an omnidirectional three-wheeled vehicle, endowed with three mechanum wheels oriented at $120^{\circ}$ one from the other to obtain the omnidirectional movement. This mobile robot has a laser scanner for its location in the working room, proximity sensors to avoid collisions and a TV camera mounted on the robot arm to visualize the objects the user wants to manipulate.

This prototype has been used to experiment and to demonstrate the capabilities of a mobile robot with these characteristics to increase the autonomy of disabled people, but its normal use is still far away due to its high cost.

Another robot developed at the SSSA in Pisa, 1992-93 is URMAUD (Unità Robotica Mobile per l'Assistenza ai Disabled). The arm has 8 degrees of freedom to provide both a higher mobility and flexibility and to facilitate its folding. The three fingered hand is endowed with tactile sensors. The manipulation of objects is complemented with a TV camera that visualizes the working area [15]. This robot is the one used in the project MOVAID (MObility and actiVity AssIstance for the Disabled) developed in the frame of TIDE (1994-97). Its goal was the integration of a complete system to be operative in a domestic environment, including not only the arm and the mobile base, but also the control of the whole assistant resources at home.

This development has tried to use all the technological resources to make possible to any kind of disabled to get a high degree of independence in the five basic environments: the kitchen, the bedroom, the living-room the study and the bath-room. Some representative robot tasks are to open the door of the refrigerator, taking some food, heating it in the microwaves and putting it on the table. The availability of a moving base allows to manipulate objects in different rooms.

\section{Conclusions}

In this chapter a survey of the evolution suffered in the field of rehabilitation, from conventional wheelchairs up to autonomous assistant robots has been presented.

The results of the works presented show that with current technological resources it is possible to develop many types of aids that can be adapted for people with different degrees of impairments. But, there is still important problems to be solved such as: power storage, that limits autonomy and forces to re-charge batteries; the miniaturization of devices, since their external aspect conditions their acceptance; and the development of more intelligent interfaces to simplify still more the use of these equipments by persons not ready to use automated equipment, and that frequently have not only motor disabilities but also visual impairment.

Cost is also a decisive factor in the acceptance of these products. The major diffusion of this technology and products and the important and increasing market for them will presumably produce a reduction of costs in the next years. 


\section{References}

[1] Amat, J. 1994 "Technology for independence" First International Conference on Robotics in Medicine, Robomed'94, Barcelona, Spain

[2] Gelin, R., Detriché, J. and Soulabaille, Y. 1994 "A navigator on a wheelchair" International Conference on Rehabilitation Robotics, ICORR'94

[3] Schraft, R. D., Wagner, J., and Schaeffer, C. 1998 "Mobility Aiding Systems" Technological Aids for Disabled, Ed. Inst. d'Estudis Catalans, Barcelona, Spain

[4] Okada, Y. And Kato, I. 1978 "Intention control of mechanical arm prosthesis" $3^{\text {rd }}$. CISM IFToMM, Symposium on Theory and Practice of Robots and Manipulators

[5] Kato, I and others. 1987 "The Waseda Hand" Internal report, Waseda University, Tokyo, Japan

[6] Rosier. J, and others. 1991 "Rehabilitation Robotics, the Manus concept" IEEE $5^{\text {th }}$ International Conference on Advanced Robotics, Pisa, Italy

[7] Hennequin, J., Platts, R., and Hennequin, Y. 1992 "Putting technology to work for the disadvantaged" Rehabilitation Robotics Newsletter, Vol. 4, N. 2

[8] Kumar, V. And Bajcsy, R 1996 "Design of customized rehabiliattion aids" $7^{\text {th }}$ International Symposium on Robotics Research. Springer, Munchen, Germany

[9] Casals, A. 1994 "Assistant arms for daily living" First International Conference on Robotics in Medicine, Robomed'94, Barcelona, Spain

[10] Casals, A., Villà, R. and Cufí, X. "Tou, an assistant arm: design and control" IEEE $6^{\text {th }}$ Int. Conference on Advanced Robotics, ICAR'93, Tokyo, Japan

[11] Jakson, R. D. 1993 "Robotics and its role in helping disabled people" Engineering Science and Educational Journal

[12] Kawamura, K. "Prospects of research on intelligent robotics systems using flexible actuators at the Intelligent robotics Lab" Research Report, Vanderbilt University

[13] Perkash, I. and others 1990 "Clinical evaluation of a vocational desktop robotics aid for severely physically disabled individuals" Report R\&D Dep. of Veterans, Palo Alto-CA

[14] Dallaway, J. L and Jakson, R. D. 1992 "RAID- a vocational robotic workstation" International Conference on Rehabilitation Robotics, Keele University, U.K

[15] Dario and others 1995 "MOVAID, a new European joint project in the field of rehabilitation robotics". $7^{\text {th }}$ Int. Conference on Advanced Robotics, Barcelona, Spain 